\title{
Food systems, diets and nutrition in the wake of COVID-19
}

\begin{abstract}
The COVID-19 pandemic is affecting food and nutrition security through economic and social systems shocks, food system disruptions and gaps in coverage of essential health and nutrition services. Food systems in low- and middle-income countries must adapt and strengthen food and nutrition security in the wake of COVID-19.
\end{abstract}

\author{
B. Carducci, E. C. Keats, M. Ruel, L. Haddad, S. J. M. Osendarp and Z. A. Bhutta
}

$\mathrm{T}$ he severe acute respiratory syndrome coronavirus 2 (SARS-CoV-2) and its mitigation responses (together referred to as COVID-19) are impacting lives and livelihoods at an unprecedented scale. Existing nutrition challenges, especially in low- and middle-income countries (LMICs), have been amplified by pandemic-related economic and food system crises that disproportionately affect the most vulnerable - women of reproductive age, young children, adolescents and the elderly $^{1,2}$. In 2019, 690 million people were undernourished, 2 billion were food insecure and 3 billion could not afford a healthy diet ${ }^{3}$; and 144 million children under-five were stunted, 47 million were wasted, 38 million were overweight and at least 340 million suffered from micronutrient deficiencies ${ }^{3}$. Projection estimates suggest that COVID-19 and the related economic recession could, by 2022 , result in an additional 9.3 million children wasted, 2.6 million stunted and 168,000 child deaths in LMICs, in the absence of appropriate response ${ }^{4}$. The World Bank estimates that COVID-19 could lead to an additional 83 to 132 million undernourished adults ${ }^{3}$ and 88 to 115 million people plunged into extreme poverty (under US\$1.90 per person per day) $)^{5}$. Forty-five countries required external food aid between April and December $2020^{6}$ and acute food insecurity stands to rise dramatically, particularly in countries currently experiencing conflict and insecurity ${ }^{6-8}$.

Food insecurity, deteriorations in diet quality, micronutrient deficiencies and other forms of malnutrition stem from fundamental, complex and dynamic changes in our food system?. Despite varying burdens and differential mitigation responses to SARS-CoV-2 across the globe, the impacts on national, regional and local food systems have consistently resulted in job losses, income shortfalls and food shortages. Rises in food waste have also been observed in some LMICs due to lower demand for perishables, limited transportation and storage capacities, and retail food price volatility ${ }^{10,11}$. These disruptions compound existing inequities in food access and expose the fragility of food systems due to conflict, weather extremes and pestilence ${ }^{7}$. Most LMICs are ill-equipped to weather the COVID-19 pandemic and its consequences, given their susceptibility to external shocks, limited financial resources and weak provision of public services.

\section{Economic and social systems shocks} As a result of COVID-19, the International Labour Organization estimates that 345 million full-time jobs were lost in the third quarter of $2020^{12}$ and the global GDP is anticipated to be $5.0-6.5 \%$ lower than predicted for 2020, while remittances are forecasted to drop in 2021 by $14 \%$ globally, triggering a recession ${ }^{13-15}$. This could impact tax revenues that fund essential public health and social security services. We expect that national and household drops in income precipitated by COVID-19 will exacerbate the vicious cycle of poverty, ill health and malnutrition in all its forms.

Food systems support the livelihoods of 1 billion people globally ${ }^{16}$. Lockdowns and restrictions at the onset of the pandemic led to closures of formal and informal food vending and food services, either temporarily or indefinitely, resulting in widespread job losses. Sudden reverse migration of the newly unemployed was triggered in some regions, exacerbating existing strains on local food and health systems - as observed in India ${ }^{17}$.

Women continue to disproportionately absorb shocks from the pandemic ${ }^{18}$; the greatest drop in income and labour force participation is observed among women, due to lower job security in the informal sector and greater childcare demands at home $^{18,19}$. Due to fear of virus transmission, lower consumer demand for goods and services, especially nutritious foods, has also resulted in more job losses in the food sector ${ }^{10,20}$. With these dramatic job and income losses, the pandemic is expected to add to the estimated 3 billion people who could not afford a high-quality diet ${ }^{3}$ before the crisis and exacerbate malnutrition; especially among women and young children who are the most nutritionally vulnerable ${ }^{19,21}$.

The loss or reduction in benefits from social protection programmes, such as food assistance and school meal programmes, will have severe consequences for the hard-to-reach and most vulnerable women, children, migrants and displaced populations who are often left out of national social protection programmes $\mathbf{s}^{18,20,22}$. According to the World Bank, in response to COVID-19, 1,414 measures were introduced, adapted or expanded within social safety-net programmes as of December 2020 in 215 countries and territories ${ }^{23}$. However, delays in implementation and the limited capacity to meet skyrocketing demand will inevitably fail to mitigate increased food insecurity among the poorest, at least temporarily ${ }^{23}$.

\section{Food system disruptions}

The pandemic has created several bottlenecks from farm to fork. Domestic supply chains consisting of micro, small and medium enterprises (SMEs), which are largely responsible for supplying food consumed in LMICs, have seen pandemic-related effects including longer lead time (due to social distancing protocols) among distributors, reduced labour capacity, increased inspections and quarantine measures, and rising operating $\operatorname{costs}^{24}$. With much of the global south depending on jobs in farming and small-scale fisheries - which are often poorly paid, unstable and unprotected, and with many employing migrant and seasonal workers (a significant proportion of which are women) $)^{19,22}-$ the shortage of labour due to COVID-19 becomes problematic during 


\section{Box 1 | Priority recommendations and actions to adapt and strengthen food systems for food and nutrition security}

1. Food systems must be made more effective, inclusive, resilient and nutritious.

- Support small- and medium-sized enterprises (including those led by women, Indigenous peoples, pastoralists, fishers and family farmers) with more effective government investments (for example, in agriculture, infrastructure and transport), policies and built-in procurement practices that support nutrient-rich foods sourced locally (including homestead and school gardens) and regionally.

- Protect and support all food system workers (formal and informal) and their rights, from future pandemics or economic shocks through social and technological innovations (for example, flexibility of labour sourcing and timing, diversifying logistics, and employee nutrition and health programmes) and appropriate personal protective equipment. This will ensure a healthy and resilient food system and will help to improve nutrition security and employment in the case of future crises, particularly for women.

- Promote shorter and more diversified food value chains, especially for nutritious, perishable foods including wild foods, animal-sourced foods and fruits and vegetables. This may result in slightly higher food prices and so governments should think of this as a worthwhile insurance against future shocks that could have irreversible effects on the youngest and most vulnerable. Also, avoid blanket export restrictions and enable safe trade corridors for nutritious foods.

- Given the suspected origins of COVID-19, there is a need to strengthen international food market transparency and to monitor food movement in real-time for data-driven decision-making around food safety compliance, food supply and food prices.
- Invest in national and community agriculture to promote the production and commercialization of nutrient-dense staples and non-staple nutritious foods.

- Invest in national or subnational micronutrient premix facilities to promote the local production of fortified staples (making sure that strict quality controls are in place) even in situations where trade is impeded. Given the reliance on non-perishable, staple foods during COVID-19, it is imperative that these foods are appropriately fortified and food processors do not have to rely solely on imported premix.

- Invest in effective behaviour change and nutrition information approaches and policies to increase the demand for nutritious foods (for example, through marketing, advertising, labelling and education).

\section{The flow of nutritious foods through social protection systems} must be increased.

- Strengthen the design and modalities of social protection programmes to enhance their ability to stimulate demand for nutritious foods. For example, link cash transfers to behaviour change to promote consumption of nutritious foods or use vouchers for nutritious foods or cash to be used in nutritious food markets.

- Ensure that social protection mechanisms are both gender-sensitive and gender-transformative by incorporating specific women's empowerment activities at individual, household and community level and direct support to women (for example, skill transfer and business training and development, microcredit and so on). This will empower women, grow businesses and provide an additional boost to food and nutrition security. peak-season times. Processing backlogs have introduced unparalleled food loss and shifts in commodity supply ${ }^{25}$. Although current forecasts suggest that staple crop production will remain relatively unaffected by the pandemic (rice, wheat, maize, lentils and soybeans), the same cannot be said for high-value, labour-intensive and perishable crops such as fresh fruits and vegetables, and animal-sourced foods ${ }^{10,25}$. This situation will add to the constraints on poor households to afford a healthy diet.

Within markets, closures and restrictions on formal (SMEs) and informal food service (that is, open-air markets, mobile vendors and cross-border traders), especially in the early stages of the pandemic, created a supply shock, while the lack of purchasing power due to employment and income losses, as well as reduced demand from SMEs, created a demand $\operatorname{shock}^{26}$. Situational analyses across Africa and Asia point to transport challenges translating into shifts in food access, food availability and in some cases, a dramatic increase in food loss, especially for nutritious, perishable foods ${ }^{10,27}$. Actual or perceived consumer concerns about food supply further affects market-seeking behaviours, such as food hoarding and panic buying, and could have devastating long-term repercussions in terms of diet quality and malnutrition $^{10,28,29}$. This includes increased consumption of cheap sources of calories (that is, starchy staples, processed cereals and unhealthy ultra-processed foods), reducing or eliminating more expensive and nutrient-rich foods, or reducing the number of meals, all of which can lead to very poor-quality diets, micronutrient deficiencies and rises in maternal and child undernutrition or overweight/obesity (especially when increased consumption of ultra-processed foods is combined with reduced physical activity, which may result from confinement and/or unemployment ${ }^{21}$. Though there are data gaps, some evidence indicates that reductions in consumption of nutrient-rich foods has been greatest for fruits and vegetables, dairy and meat in both high-income and LMIC contexts ${ }^{30-32}$.

\section{Gaps in essential health services} Although empirical country- and intervention-specific evidence is still unavailable, the COVID-19 pandemic has undoubtedly created significant gaps in health programming and service delivery across the globe - including routine and essential services for reproductive, maternal, neonatal, child and adolescent health and nutrition $^{33}$. At the onset of the pandemic, the demand for health facility and community health services plummeted in several countries due to virus transmission concerns, travel restrictions, and income losses $^{33}$. Reductions in community healthcare worker visits, disrupted community screening and management of acute malnutrition, and diversion of available health workers to COVID19-related activities, have led to lower coverage of nutrition counselling, including for breastfeeding promotion and infant and young child feeding support ${ }^{3}$. Drops in the provision and uptake of vitamin-A supplementation and immunization programmes, and services for the prevention and treatment of infections and severe acute malnutrition, could precipitate dramatic increases in child mortality ${ }^{33}$. Disruptions to imports, local production and distribution of essential preventive and therapeutic nutritional products (such as multiple 
micronutrient supplements and oral rehydration salts) have also been severe ${ }^{34}$. Lags in the availability of micronutrient premix and other essential inputs needed for staple food fortification have slowed processing times ${ }^{27}$. Notably, we have not captured the full extent of country or local COVID-19 responses, such as investments and innovations that might adapt and restore services. Until the data is available and interpretable, essential health and nutrition services coverage information in the wake of COVID-19 relies on fragmented narratives.

\section{Making a difference through transformation}

Since the beginning of the twenty-first century, discourse on a global food system transformation that prioritizes healthy diets and nutrition has been echoed throughout agencies and among scientists alike ${ }^{35}$. The COVID-19 pandemic has exposed vulnerabilities within the food system and emphasized the need for a holistic systems approach to hasten united efforts towards our global targets and goals. The 2021 Food Systems Summit provides an opportunity to rethink food systems and design long-term planetary health reforms to build resilient communities, protect rural agrarian populations and promote sustainable supply chains and food safety.

We propose two specific and urgent recommendations (Box 1) to ensure that high-quality diets and nutrition are prioritized in mitigating the long-term effects of the pandemic, and any comparable future crises, through sustainable reforms. Existing inequities within food systems, especially those related to gender and otherwise marginalized populations, must be addressed through policy responses and social protection programmes to improve the affordability of healthy diets and prevent both current and intergenerational malnutrition. Leveraging and engaging with national and local governmental actors, agribusiness, donors and developmental partners, and the international community to translate these recommendations is key to their implementation ${ }^{8,36-39}$.
B. Carducci (D) 1,2, E. C. Keats ${ }^{1}$, M. Ruel(D) ${ }^{3}$, L. Haddad ${ }^{4}$, S. J. M. Osendarp (D) ${ }^{5}$ and Z. A. Bhutta (D) 1,2,6凶 ${ }^{1}$ Centre for Global Child Health, Hospital for Sick Children, Peter Gilgan Centre for Research, and Learning (PGCRL), Toronto, Ontario, Canada. ${ }^{2}$ Department of Nutritional Sciences, University of Toronto, Faculty of Medicine, University of Toronto, Toronto, Ontario, Canada. ${ }^{3}$ International Food Policy Research Institute, Washington DC, USA. ${ }^{4}$ Global Alliance for Improved Nutrition, Geneva, Switzerland. ${ }^{5}$ Micronutrient Forum, Washington, DC, USA. ${ }^{6}$ Institute for Global Health \& Development, Aga Khan University South-Central Asia, East Africa and United Kingdom, Karachi, Pakistan.

$\bigotimes_{\text {e-mail: zulfiqar.bhutta@sickkids.ca }}$

Published online: 18 February 2021

https://doi.org/10.1038/s43016-021-00233-9

References

1. Akseer, N., Kandru, G., Keats, E. C. \& Bhutta, Z. A. Am. J. Clin. Nutr. 112, 251-256 (2020).

2. Laborde, D., Martin, W. \& Vos, R. in COVID-19 \& Global Food Security (eds Swinnen, J. \& McDermott, J.) Ch. 2, 16-19 (International Food Policy Research Institute, 2020); https://www. ifpri.org/interactive/covid

3. The State of Food Security and Nutrition in the World 2020: Transforming Food Systems for Affordable Healthy Diets (FAO, IFAD, UNICEF, WFP and WHO, 2020); http://go.nature. com/3jzMGAP

4. Osendarp, S. et al. Preprint at Research Square https://doi. org/10.21203/rs.3.rs-123716/v1 (2020).

5. Poverty and Shared Prosperity: Reversals of Fortune (World Bank Group, 2020); https://go.nature.com/3jv6uFG

6. Forecasting Threats to the Food Chain Affecting Food Security in Countries and Regions (FAO, 2020); https://go.nature. com/3cRZ7Xy

7. Global Report on Food Crises: Joint Analysis for Better Decisions (Food Security Information Network and Global Network Against Food Crises, 2020); http://go.nature.com/3a0Ikzm

8. Cable, J., Jaykus, L.-A., Hoelzer, K., Newton, J. \& Torero, M Ann. N. Y. Acad. Sci. 1484, 3-8 (2020).

9. 2020 Global Nutrition Report: Action on Equity to End Malnutrition (Global Nutrition Report, 2020); http://go.nature com/39YM5FR

10. Policy Brief: The Impact of COVID-19 on Food Security and Nutrition (United Nations, 2020); http://go.nature.com/3plGNgV

11. Food Security and COVID-19 (World Bank Group, 2020); http://go.nature.com/3aKfJh1

12. ILO Monitor: COVID-19 and the World of Work 6th edn (International Labour Organization, 23 September 2020); https://go.nature.com/3juQ2Vz

13. World Economic Outlook Update, June 2020 (International Monetary Fund, 2020); http://go.nature.com/34bvYSC

14. Global Economic Prospects (World Bank Group, 2020); http://go.nature.com/3cQIY4l

15. COVID-19: remittance flows to shrink $14 \%$ by 2021. World Bank Group (29 October 2020); http://go.nature.com/3jvjhYk

16. Mbow, C. et al. in Climate Change and Land: An IPCC Special Report on Climate Change, Desertification, Land Degradation, Sustainable Land Management, Food Security, and Greenhouse
Gas Fluxes in Terrestrial Ecosystems (eds Shukla, P. R. et al.) Ch. 5 (IPCC, 2019).

17. COVID-19 Crisis Through a Migration Lens (World Bank Group, 2020); https://go.nature.com/3pZKiWD

18. Policy Brief: The Impact of COVID-19 on Women (United Nations, 2020); https://go.nature.com/3p6lJ9e

19. Montalvao, J. \& Van de Velde, P. COVID-19 and Food Security: Gendered Dimensions (The World Bank, 2020); https://go.nature. com/2MARudl

20. WFP Global Response to COVID-19: June 2020 (World Food Programme, 2020); https://go.nature.com/3q3I0ph

21. Headey, D. \& Ruel, M. in COVID-19 \& Global Food Security (eds Swinnen, J. \& McDermott, J.) Ch. 8, 38-41 (International Food Policy Research Institute, 2020); https://www.ifpri.org/ interactive/covid

22. Hidrobo, M., Kumar, N., Palermo, T., Peterman, A. \& Roy, S. in COVID-19 \& Global Food Security (eds Swinnen, J. \& McDermott, J.) Ch. 21, 91-96 (International Food Policy Research Institute, 2020); https://www.ifpri.org/interactive/covid

23. Gentilini, U. et al. Social Protection and Jobs Responses to COVID-19: A Real-Time Review of Country Measures (World Bank Group, 2020); https://go.nature.com/3mRDxUz

24. Reardon, T. \& Swinnen, J. in COVID-19 \& Global Food Security (eds Swinnen, J. \& McDermott, J.) Ch. 30, 132-136 (International Food Policy Research Institute, 2020); https://www.ifpri.org/ interactive/covid

25. Sustainable Crop Production and COVID-19 (FAO, 2020); http://go.nature.com/3aJKa75

26. Torero M. in COVID-19 \& Global Food Security (eds Swinnen, J. \& McDermott, J.) Ch. 27, 118-122 (International Food Policy Research Institute, 2020); https://www.ifpri.org/interactive/covid

27. Impact of COVID-19 on Food Systems: A Situation Report (Global Alliance for Improved Nutrition, 2020); https://go.nature. com/3qcznso

28. Wang, E., An, N., Gao, Z., Kiprop, E. \& Geng, X. Food Secur. 12, 739-747 (2020).

29. Benker, B. Appetite 156, 104981 (2021)

30. Tesfaye, A., Habte, Y. \& Minten, B. in COVID-19 \& Global Food Security (eds Swinnen, J. \& McDermott, J.) Ch. 9, 42-45 (International Food Policy Research Institute, 2020); https://www. ifpri.org/interactive/covid

31. Di Renzo, L. et al. J. Transl. Med. 18, 229 (2020).

32. Jayawardena, R. \& Misra, A. Diabetes Metab. Syndr. 14, 1085-1086 (2020).

33. Pulse Survey on Continuity of Essential Health Services During the COVID-19 Pandemic (World Health Organization, 2020): http://go.nature.com/3jtva12

34. McDonnell, A., Chalkidou, K., Yadav, P. \& Rosen, D. Understanding the Impact Of COVID-19 On Essential Medicine Supply Chains (Center for Global Development, 2020); http://go.nature.com/39XkJzz

35. Branca, F. et al. BMJ 364, 1296 (2019).

36. Jepsen, S. D. et al. J. Agromedicine 25, 417-422 (2020).

37. Perdana, T., Chaerani, D., Achmad, A. L. H. \& Hermiatin, F. R. Heliyon 6, e05128 (2020).

38. Heck, S. et al. Food Secur. 12, 823-830 (2020).

39. Patterson, G. T., Thomas, L. F., Coyne, L. A. \& Rushton, J Glob. Food Sec. 26, 100424 (2020).

\section{Author contributions}

All authors contributed to writing, reviewing and revising the manuscript. All authors approved the final manuscript as submitted and agreed to be accountable for all aspects of the work.

Competing interests

The authors declare no competing interests. 